\title{
Triazene Derivative TriN2755
}

National Cancer Institute

\section{Source}

National Cancer Institute. Triazene Derivative TriN2755. NCI Thesaurus. Code C95208.

A synthetic triazene derivative with antineoplastic activity. Upon metabolic activation via $\mathrm{N}$-demethylation, TriN2755 is converted into highly reactive carbocations that can alkylate DNA and other macromolecules, thereby resulting in DNA cross links, inhibiting DNA replication and repair, and subsequently inducing apoptosis. This agent has high hydrophilicity and photostability and shows a favorable toxicity profile over the other triazenes. 